\title{
Dilemas éticos en la práctica clínica en las unidades de cuidados intensivos y paros cardiorrespiratorios
}

Ethical dilemmas in clinical practice in the intensive care units and cardiorespiratory arrests

\section{Oscar Alberto Castejón Cruz, ${ }^{1}$ Helga Leticia Hernández Pacheco, ${ }^{2}$ René Alexander Núñez Savoff, ${ }^{3}$ ShannieWaleska Bush Wood. ${ }^{3}$}

\author{
`Médico General, Máster en Dirección Estratégica de Organizaciones de Salud; Centro de Evaluaciones Médicas y Psicológicas; Tegucigalpa; \\ ${ }^{2}$ Médico General, Traumacentro; Comayagüela; \\ ${ }^{3}$ Médico General, Wood Medical Center; Roatán, Islas de la Bahía; Honduras.
}

\begin{abstract}
RESUMEN. Antecedentes. La bioética entiende que toda propuesta terapéutica o diagnóstica debe definir la meta por lograr, el provecho por obtener y los riesgos por enfrentar. Requiere conocer la historia natural de la enfermedad y sus complicaciones, así como la estimación de las posibilidades de éxito y fracaso ajustadas a cada paciente en particular. Fuentes: búsqueda bibliográfica en Medline, ProQuest, SCOPUS, usando las palabras claves "ética en el acto quirúrgico" "bioética" "ética clínica" "ética en obstetricia "aborto terapéutico", "consideraciones éticas en el embarazo y cáncer" "ética y cirugía oncológica" "dilemas éticos en la práctica anestésica", tanto en castellano e inglés, limitada al periodo 2014- 2018. Los modelos más citados son los incluidos en la presente revisión. Desarrollo: El comportamiento ético no sólo debe estar limitado al acto quirúrgico, sino a toda la conducta del cirujano, aceptando que sus actos deben ser éticamente válidos, desde el estudio clínico del paciente y solicitud de estudios auxiliares de diagnóstico basados en un criterio razonado hasta la información otorgada al paciente y familiar, el acto quirúrgico y vigilancia postoperatoria Conclusiones: Cada día nos enfrentamos a dilemas éticos por lo que es evidente la necesidad de capacitación del personal de salud tanto médico, enfermería y todo personal que tiene contacto con paciente en aras de evitar el ensañamiento terapéutico y lograr el máximo bienestar del enfermo y sus familiares, desde un punto de vista integral. La principal e inicial obligación ética de todo médico es estar preparado.

Palabras clave: Cirugía, Ética, Ética clínica, Ginecología, Obstetricia, Procedimientos innecesarios.
\end{abstract}

\section{INTRODUCCIÓN}

Los actos médicos son resultado de una serie de eventos sucesivos que abarcan desde diagnóstico, pronóstico y tratamiento, hasta la implementación y evaluación de resultados. En cada etapa del proceso se presentan al médico múltiples alternativas de acción; su elección se fundamenta en juicios de valor, donde se opta por la alternativa que resulta "mejor". Para llegar a esta decisión se ponderan los más variados elementos, como factores técnicos, económicos, éticos, emocionales y prácticos. ${ }^{1}$

El ejercicio de la medicina ha planteado siempre problemas éticos y ha exigido del médico una elevada calidad moral. Sin embargo, ninguna época como la nuestra ha planteado tantos y tan complejos problemas morales a los médicos, y nunca como ahora se ha requerido de una adecuada formación ética de los profesionales sanitarios. Las razones de este cambio son fundamentalmente: el enorme progreso de la tecnología sanitaria, el fenómeno de emancipación de los pacientes y su mayor protagonismo en el proceso de toma de decisiones, y la distribución equitativa de recursos económicos limitados y escasos. ${ }^{2}$

Recibido 4-3-2018; aceptado para publicación 4-11-2018

Dirigir correspondencia a: Dr. Óscar Alberto Castejón Cruz

Correo electrónico: oacastejon@gmail.com

Conflictos de interés. Los autores declaramos no poseer conflictos de interés en relación a este artículo.

DOI: https://doi.org/10.5377/rmh.v87i1.11936
La medicina, por su propia naturaleza, es un quehacer moral. El médico y otros profesionales de la salud dirigen toda actividad a tratar y cuidar seres humanos, y sus decisiones intentan devolver la salud al paciente, curarlos de sus dolencias, prevenirlos de enfermedades y al mismo tiempo deben poseer gran carga de humanidad, respetando la integridad personal del hombre necesitado de ayuda. Para asumir esta responsabilidad y hacer frente a los problemas que se les plantean, no basta sólo una constante formación científica, sino también sensibilidad y conciencia ética para identificar problemas y buscar soluciones racionales. $^{3}$

Dilema es esencialmente la duda o disyuntiva entre dos cosas; dilema ético aquel que compete a la naturaleza moral, es decir, que plantea cómo obrar bien, cómo evitar hacer daño, valorar las consecuencias y lograr lo mejor para la mayoría de las personas involucradas en una situación observando reglas morales. Como menciona Rushworth Kidder un dilema ético no implica tener que elegir entre lo correcto y lo incorrecto sino, elegir entre dos cosas correctas. ${ }^{4}$ Los principios básicos de bioética son aquellos criterios generales que sirven como punto de apoyo para justificar los preceptos éticos y valoraciones de las acciones humanas en el campo biosanitario. Uno de los principales objetivos de la bioética es facilitar al profesional sanitario e investigador la toma de decisiones racionales concretas en resolución de conflictos de valores planteados en su actividad. En la relación médico-paciente, la toma de decisiones 
ante una situación clínica, dependerá de las valoraciones morales que hagan los componentes de la relación, que pueden no coincidir. Es principio que los clínicos actuemos siempre teniendo en cuenta los 4 principios fundamentales que definen la práctica clínica ética: beneficencia, no maleficencia, autonomía y justicia, que deben considerarse como los principios generales de la Bioética, no sólo en el ámbito de la experimentación humana, en el cual se habían definido, sino en cualquier ámbito de la biomedicina en el que se tengan que resolver problemas prácticos concretos en una sociedad pluralista. ${ }^{3}$ Con el objetivo de analizar estos aspectos se realizó una revisión bibliográfica y se hizo un análisis crítico del tema y contribuir a la discusión para la resolución de los dilemas ético-clínicos que no niega la subjetividad de los actores involucrados, sino que la incluye de una manera objetiva. ${ }^{1}$

\section{DILEMAS ÉTICOS EN LA UNIDAD DE CUIDADOS IN- TENSIVOS (UCI) DEL ADULTO Y PACIENTES EN ESTADO TERMINAL}

En las Unidades de Cuidados Intensivos (UCl) se plantean a diario una serie de problemas muy particulares del trabajo en ellas en relación con la aplicación de los principios bioéticos derivados de la aplicación de métodos diagnósticos y terapéuticos invasivos, de la frecuente incompetencia del paciente, de la comprensión de lo que es o no fútil o la necesidad de abordar la existencia del límite en la atención médica o del esfuerzo terapéutico. ${ }^{4}$ La reflexión ética sobre la situación terminal de una enfermedad y la orden de no reanimación cardiopulmonar, como forma de adecuación del esfuerzo terapéutico, son temas fundamentales para comprender en profundidad a los enfermos que se encuentran en esa fase terminal. Hoy, con nuevos conocimientos, valores éticos y recursos científicos tecnológicos, usados adecuadamente, permiten marchar hacia una nueva etapa histórica de justicia y humanismo, donde las decisiones médicas tengan en cuenta no solo los conocimientos científicos, si no también lo humano. ${ }^{5}$

El descubrimiento y aplicación de nuevos conocimientos científicos y técnicos en el terreno de la medicina, especialmente en los temas de resucitación cardiopulmonar, los avances logrados en el conocimiento de la fisiología cardiaca y respiratoria, logran todos en su conjunto prolongar la vida de los enfermos por tiempo indefinido. ${ }^{3}$ Todos ellos fueron peldaños que condujeron a la generalización de una nueva modalidad de atención médica, donde el sostén de las funciones vitales permite intervenir en los procesos de la vida y la muerte; tal es el caso de la prolongación artificial de la vida mediante instauración y mantenimiento de medidas de soporte vital. ${ }^{5}$

En las últimas décadas los avances tecnológicos han provocado un aumento en la sobrevida de pacientes considerados previamente irrecuperables, dando lugar a una prolongación del proceso de morir, tratando de conservar la vida. Los cuidados al final de la vida son un importante componente en las unidades de cuidados intensivos pediátricos como en adultos. ${ }^{6}$ El objetivo principal del equipo de salud en la unidad de cuidados intensivos (UCl) es restaurar la salud (salvar la vida) de los pacientes en estado crítico, con padecimientos que tienen posibilidad de recuperación y requiriendo de medidas especializadas de soporte multiorgánico administradas por profesionistas especializados. ${ }^{7}$ La orden de no reanimación cardiopulmonar y cerebral (No RCP-C), es una forma de adecuación del esfuerzo terapéutico, reconocida por muchos países. ${ }^{5}$ En Honduras, no contamos con legislación al respecto por lo tanto el soporte vital depende del criterio del médico tratante pero no impide que al paciente se le proporcionen otros tratamientos o cuidados ordinarios, adecuados y proporcionales a su situación clínica real.

En las unidades de atención al paciente grave y en estado crítico, debemos establecer procedimientos que aseguren que no se resucitará a los pacientes a los cuales no debe resucitarse, por su situación de terminalidad. Se puede puntualizar lo esencial del concepto de enfermedad terminal, reconociéndole cinco características establecidas por la OMS y citadas por el Dr. C. Ricardo Hodelín, especificada en su artículo, ${ }^{8}$ descritas a continuación: 1) Presencia de una enfermedad avanzada, progresiva, incurable; 2) Falta de posibilidades razonables de respuesta al tratamiento específico; 3) Presencia de numerosos problemas o síntomas intensos, múltiples, multifactoriales y cambiantes; 4) Gran impacto emocional en paciente, familia y equipo terapéutico, muy relacionado con la presencia explícita o no, de la muerte; 5) Pronóstico de vida inferior a seis meses.

En la toma de decisiones en el paciente crítico al final de la vida, el médico debe tener en cuenta la lex artis constituida por los principios científicos y éticos que orientan la práctica médica. ${ }^{9}$ La limitación del soporte de vida (LSV), también denominada limitación del esfuerzo terapéutico es definida como la decisión de no establecer un tratamiento médico o de interrumpir un soporte avanzado de vida ya establecido en pacientes terminales sin posibilidad de recuperación. ${ }^{10}$ Sin embargo, es ético que los médicos se nieguen a proporcionar un tratamiento médico inapropiado o inútil. Comprender la justificación de la futilidad puede ser relevante para decidir la forma más adecuada de resolver las disputas. Desde el punto de vista legal no existen políticas sobre la futilidad ni en EE.UU. ni en Honduras, y si las hay, se usan poco. En general, si la familia lo solicita, el tratamiento es usualmente continuado. Un estudio sobre limitación de tratamientos de soporte vital tras el ingreso en UCl, objetivó que la futilidad, el pronóstico y la pobre calidad de vida son las diferentes razones para limitar tratamientos. ${ }^{11}$

\section{Modelos para la toma de decisiones de limitación del esfuerzo terapéutico (LET)}

Existen en la literatura médica propuestas de modelos de toma de decisiones éticas en escenarios de sobre la limitación del esfuerzo terapéutico. El planteado por Reyes, ${ }^{12}$ que parte de dos tipos de pacientes: los plenamente autónomos y los que cuentan con una autonomía muy reducida o inexistente; en pacientes con autonomía muy limitada o inexistente se debe indagar por la existencia de algún documento de voluntades anticipadas vigente suscrito por el propio paciente. Si no existe tal documento, el punto de partida del proceso que puede llevar a tomar una decisión de LET suele tener una indicación médica 
basada en consideraciones acerca de la futilidad de actuaciones médicas posibles, en el sentido de que se dispone de los apropiados recursos tecnológicos, pero que con toda probabilidad no podrán revertir el proceso mórbido que afecta al paciente. ${ }^{12,13} \mathrm{El}$ modelo de Simón Lorda pretende articular los dos momentos de las decisiones clínicas: la indicación médica de realización de la LET con la autorización del paciente para su realización. ${ }^{12,14,15}$

\section{Principios éticos en el Paro Cardiorrespiratorio (RCP)}

El consenso internacional sobre paro cardíaco, conocido como 《estilo Utstein》, define el paro como el cese de la actividad mecánica cardiaca, confirmado por la ausencia de conciencia, pulso detectable y respiración (o respiración agónica entrecortada) ${ }^{16}$. La muerte súbita cardíaca se define como la que ocurre de modo inesperado, dentro de la primera hora del comienzo de los síntomas, en pacientes cuya situación previa no hacía previsible un desenlace fatal. Muerte súbita y paro cardiorrespiratorio (PCR) suelen usarse como sinónimos. Ambos son conceptos de limites arbitrariamente establecidos en torno a un mismo fenómeno. En este sentido, es importante la realización de una buena historia clínica con todos los antecedentes personales de interés y el motivo de ingreso y, además, anotar si el paciente no fuera reanimable (ordenes de "no-RCP"). Esto facilitaría enormemente la decisión de empezar o no la RCP, ayudaría a aclarar la causa de la PCR y que el médico responsable de la reanimación se centrara exclusivamente en ella en ese momento.

Es esencial señalar que el objetivo de la RCP no es la mera restauración de las funciones vitales, sino la supervivencia con un mínimo de calidad de vida. El reanimar a una persona con un paro cardiorrespiratorio reconoce el valor de la vida cuando se está frente a una situación vitalmente limite que se estima reversible. En estos casos, debe considerarse una obligación médica y ética intentar la reanimación. Sin embargo, la aplicación indiscriminada de tal maniobra implica, también el riesgo de menoscabar la dignidad del morir y de dejar a los pacientes con graves secuelas neurológicas. Las decisiones en la RCP deben estar basadas en los principios básicos de la ética. Las situaciones de emergencia vital hacen más difícil la toma de decisiones. En los menores de edad son determinadas por sus padres y/o tutores considerando el mejor interés del menor. Existen situaciones en las que está claramente indicado el inicio de la RCP y otras en las que está justificado suspender las maniobras. Otros aspectos éticos incluyen: posibles donantes y el aprendizaje de maniobras. ${ }^{17}$

\section{DILEMAS ÉTICOS EN LA UNIDAD DE CUIDADOS IN- TENSIVOS PEDIÁTRICOS (UCIP)}

Con el avance de la tecnología e innovadores enfoques en el diagnóstico y tratamiento, se ha logrado la recuperación de muchos niños con enfermedades antes consideradas mortales. Estas innovaciones han generado diversos beneficios para la salud; no obstante, plantean una serie de dilemas éticos frente al cuidado del paciente pediátrico. Esto debido a que no todo lo técnicamente posible puede ser siempre éticamente justificable. ${ }^{18}$ Los niños que ingresan a estas unidades requieren la aplicación de medidas de soporte vital, debido a su patología. Son enfermos críticos, con amenaza grave para su vida, potencialmente recuperables y que necesitan vigilancia continua de sus funciones vitales. En estos casos, los objetivos de las unidades son: mantener la calidad de vida, aliviar el sufrimiento, evitar los riesgos para el paciente, restaurar la salud y respetar los derechos del paciente. Por este motivo, las actividades se basan principalmente en la aplicación de dos modelos de atención: el curativo y el paliativo. ${ }^{18}$ Los problemas éticos fundamentales se relacionan con algunos conceptos surgidos de la práctica médica moderna: la eutanasia, la ortotanasia y la distanasia. ${ }^{19}$ El dilema ético radica en que la eutanasia no es aceptada por la mayoría de las legislaciones. Dar el derecho al médico de quitar la vida, en cualquier forma, sería ir en contra de lo natural, prima el respeto por la vida, en cualquiera de sus variantes. Así lo estableció hace casi 25 siglos el Juramento Hipocrático, por lo que la eutanasia involuntaria es inaceptable. ${ }^{18,19}$ La ortotanasia, nombrada también como limitación del esfuerzo terapéutico (LET), es definida como la decisión mediata sobre la no implementación o la retirada de tratamientos médicos, al anticipar que no conllevarán un beneficio significativo al paciente, tal como se discutió previamente. ${ }^{19,20}$

No hay que confundir la eutanasia con la ortotanasia o LET. En la primera, la idea que prevalece en la mente del que la aplica es poner fin a la vida del enfermo por considerar que se pone fin a un sufrimiento; en la LET en cambio, la idea fundamental es no poner en práctica un tratamiento determinado que se sabe con seguridad que no va a resultar en ningún beneficio, sino que producirá dolor, sufrimiento y prolongación de la agonía. ${ }^{19}$ El término contrario a la ortotanasia es la distanasia (obstinación terapéutica o encarnizamiento terapéutico), del griego dis 'mal, algo mal hecho', y thanatos 'muerte'. Significa retrasar la muerte todo lo posible, por todos los medios, proporcionados o no, aunque no haya esperanza alguna de curación y aunque eso signifique infligir al moribundo unos sufrimientos sobreañadidos. ${ }^{3,9,19}$

Las estrategias para el manejo del niño gravemente enfermo atendido en una UCIP desde una perspectiva bioética, descritas en la literatura, se pueden agrupar en cuatro ejes principales, ${ }^{18}$ descritos a continuación. La primera estrategia consiste en considerar la limitación del esfuerzo terapéutico como una decisión ético-clínica, ya que esta debe plantearse cuando las alteraciones consecuentes a una enfermedad o las complicaciones que surjan en el curso de la evolución de algún cuadro patológico puedan determinar la inconveniencia del mantenimiento de medidas de soporte vital, y cuando es inútil continuar el tratamiento en pacientes sin ninguna posibilidad de recuperación de una vida humana digna. La segunda estrategia consiste en incorporar la toma de decisiones éticas como un proceso, ya que el niño posee dignidad como sujeto, con derechos propios que no deben ser menospreciados ni menos irrespetados. Por este motivo, el pediatra siempre debe respetar la vida humana, evitando incurrir en extremos peligrosos relacionados con el 
manejo del niño gravemente enfermo, que lo lleven a la futilidad o encarnizamiento terapéutico para prolongar su vida biológica 0 al desinterés en su atención, por las consecuencias negativas que puede ocasionar en la familia. En las UCIP, la decisión de limitar el esfuerzo terapéutico puede surgir del equipo médico, de los padres o tutores del niño afectado o del propio paciente, en el caso de adolescentes responsables.

La tercera estrategia consiste en reconocer al paciente y familia como centro del proceso de toma de decisiones éticas, porque se debe procurar que los padres del niño logren una adecuada comprensión de la situación médica del niño. Una vez que los padres tengan ese nivel de comprensión, es el médico quien debe tomar la iniciativa para plantear la posibilidad de limitar los tratamientos, ofrecer a los padres las razones, tanto médicas como éticas, y darles el tiempo necesario para que ellos reflexionen y lleguen a una conclusión.

La cuarta estrategia consiste en disponer de un Comité de Ética Asistencial como apoyo en la toma de decisiones, ya que estos se constituyeron por la necesidad de resolver conflictos respecto del curso de acción terapéutico que involucre la vida de una persona. Se sugiere ampliar la información sobre estos comités, de modo de profundizar sobre su constitución y su importancia en el apoyo de la toma de decisiones en la práctica médica, destacando la importancia de este grupo multidisciplinario integrado al quehacer del equipo de salud, y su rol fundamental en las resoluciones, especialmente cuando se trata de los dilemas del final de la vida humana.

\section{Criterios locales de no ingreso a UCl}

Con estos criterios ${ }^{21}$ cada paciente es analizado individualmente en su contexto: 1) Paciente terminal; 2) Paciente en estado vegetativo permanente o estado de mínima conciencia; 3) Paciente fuera de alcance médico-quirúrgico en consenso con sus médicos tratantes, independiente del sistema u órgano involucrado. ${ }^{21}$ En las unidades de cuidados intensivos pediátricos existe el confort care neonatal, dado a aquellos bebes que nacen con bajísimas posibilidades de vida y fallecen a las horas o a los pocos días. Estos niños presentan los mismos derechos de los que se encuentran en estado sano, cuando en la madre; en sus controles prenatales; salta a relucir que presenta alguna malformación con pronóstico reservado de vida, por lo que se requiere de un tratamiento adecuado, basado en un protocolo, para brindar una buena atención perinatal. Los padres deben trabajar con el equipo médico para garantizar los cuidados básicos que reclaman estos bebés y hacerles su vida confortable sin perder su dignidad y grandeza que todo ser humano merece por sí mismo. ${ }^{22}$

\section{CONCLUSIÓN}

Una adecuada relación médico-paciente-familia, una correcta formación ética del personal de la salud, así como la continua investigación minuciosa, constituyen la clave que resultará en estrategias dirigidas a resolver los dilemas éticos que aparecen en la atención de los pacientes en nuestra practica medica diaria. Cada día nos enfrentamos a dilemas éticos por lo que es evidente la necesidad de capacitarnos continuamente en estos temas al personal de salud tanto médico, enfermería y todo personal que tiene contacto con paciente en aras de evitar el ensañamiento terapéutico y lograr el máximo bienestar del enfermo y sus familiares, desde un punto de vista integral.

El profesional de la salud que está integralmente formado debe ser capaz de entender el contexto del paciente $y$, dentro de su ética, involucrar a la familia en la toma de decisiones. También es de gran importancia la participación del personal de enfermería, no solo en la discusión junto con los médicos del servicio y en la información a los familiares, sino también como iniciadores del proceso de toma de decisiones, pues la percepción que tienen del enfermo y su entorno (familia, condicionantes sociales, hábitos, etc.) enriquece, sin duda, la visión de la situación clínica del paciente. La ocurrencia de conflictos éticos en la Unidad de Cuidados Intensivos es muy frecuente, lo que evidencia que los profesionales enfrentan situaciones difíciles que podrían ser facilitadas o subsanadas si son adecuadamente entrenados y formados en Bioética. No cabe duda que, aunque la ética no aporte las respuestas definitivas es muy útil para conocer que supone tomar la decisión y elaborar una metodología de abordaje de los dilemas morales que facilite ser consistente en las respuestas que se proponen; el elemento básico de la formación clínica en urgencias es el aprendizaje en la toma de decisiones racionales, tanto de tipo técnico como ético. Consideramos que este tema constituye un aspecto más de una correcta y actualizada formación del médico.

CONTRIBUCIONES: OC concibió la idea original de escribir el presente artículo y también lideró el desarrollo del mismo. $\mathrm{HH}, \mathrm{RN}$ y SB apoyaron en la búsqueda bibliográfica e incorporación de comentarios. Todos los autores aprobaron la versión final artículo.

\section{REFERENCIAS}

1. López Barreda R. Modelos de análisis de casos en ética clínica. Acta Bioet. 2015;21(2):281-90.

2. Cisneros Sánchez LG, Carranza Garcés E, Martinez Peró RM. Cuestiones éticas relacionadas con la atención a los pacientes con insuficiencia cardíaca terminal. CorSalud. 2017;9(1):23-9.

3. Díaz Hernández D. Conocimientos y actitudes sobre limitación del esfuerzo terapéutico en el equipo interdisciplinar de cuidados intensivos. [Tesis]. España: Universidad La Laguna. Facultad de Ciencias de la Salud. Enfer- mería y Fisioterapia; 2016.

4. Alonso Mariño AL, Alonso Mariño OL, López Rodríguez EF. Dilemas éticos de las decisiones médicas en cuidados intensivos. Acta Méd Cent. 2014;8(1):37-45.

5. Betancourt G, Betancourt Reyes GL. La Orden no reanimación cardiopulmonar cerebral en la adecuación del esfuerzo terapéutico. Rev Cuba Cardiol Cir Cardiovasc. 2017;23(1):240-249.

6. Morales Valdés G, Alvarado Romero T, Zuleta Castro R. Limitación del es- 
fuerzo terapéutico en cuidados intensivos pediátricos: conocimiento y actitudes bioéticas del profesional médico. Rev Chil Pediatr. 2016;87(2):116120

7. Kaneko-Wada FdJT, Domínguez-Cherit G, Colmenares-Vásquez AM, Santana-Martínez P, Gutiérrez-Mejía J, Arroliga AC. El proceso de muerte en la unidad de cuidados intensivos (UCl). Punto de vista médico, tanatológico y legislativo. Gac Med Mex. 2015;151(5):628-34.

8. Hodelín Tablada R. El principio del doble efecto en la sedación a pacientes terminales. MEDISAN. 2012;16(6):949-59.

9. Pérez Pérez FM. Adecuación del esfuerzo terapéutico, una estrategia al final de la vida. Semergen. 2016;42(8):566-574.

10. Dias Araujo Nunes EC, de Oliverira Sousa J. Limitación del soporte de vida en la Terapia Intensiva: Percepción médica. Rev bioét. 2017; 25 (3): 55462.

11. Grinberg AR, Tripodoro VA. Futilidad médica y obstinación familiar en terapia intensiva ¿Hasta cuándo seguir y cuándo parar? MEDICINA (Buenos Aires) 2017; 77(6): 491-496.

12. Rincón Roncancio M, Garzón Díaz F. Problemas Éticos del retiro o limitación de tratamiento vital en unidades de cuidados intensivos. Rev Latinoam Bioét. 2015;15(2):42-51.

13. Royes A. Bioética y práctica clínica: propuesta de una guía para la toma de decisiones de limitación del esfuerzo terapéuticos. Revista de Calidad Asistencial. 2005;20(7):391-395

14. Simón Lorda P, Esteban López MS, Cruz Piqueras M. Limitación del esfuerzo terapéutico en cuidados intensivos: Recomendaciones para la elaboración de protocolos. España: Junta de Andalucía; 2014.88 p.
15. Gherardi CR, Biancolini C, Butera JJ, Calvillo L, Canteli MM, Cardonnet I, et al. Pautas y recomendaciones para la abstención y/o retiro de los métodos de soporte vital en el paciente crítico. Red Inst. 1999;16(2):53-56.

16. Vera Carrasco O. Conducta ética en el paro cardiorrespiratorio. Rev Médica. La Paz. 2016;22(1):69-79.

17. Avilés-Martínez KI, López-Enríquez A, García-Armenta MB, Pérez-Díaz Cl, Jiménez-Pérez BA, Palacios-Márquez $\mathrm{R}$, et al. Toma de decisiones para iniciar la reanimación cardiopulmonar. Rev Médica MD. 2017;8(4):165170.

18. Pino Armijo P, San Juan Hurtado L, Monasterio Ocares MC. Implicancias éticas en el manejo del niño gravemente enfermo atendido en una unidad de paciente crítico pediátrica. Acta bioeth. 2014;20(1):51-59.

19. Uriarte Mendez AE, Vila Diaz J, Pomares Perez Y, Prieto Apesteguia A, Capote Padron JI. La familia y la limitación del esfuerzo terapéutico en los cuidados intensivos pediátricos. Aspectos éticos. Medisur. 2014;12(1):16-23

20. Betancourt Betancourt GJ, Rivero Castillo JA. Limitacion del esfuerzo terapeutico: aspectos religiosos y culturales. Rev Hum Med. 2015;15(1):145162.

21. von Dessauer Grote B, Benavente Eugenia C, Monje Lara E, Bongain Acevedo J, Ordenes Duffau N. Limitación de tratamiento de soporte vital en una Unidad de Cuidado Intensivo pediátrica chilena: 2004-2014. Rev Chil Pediatr. 2017;88(06): 751-758.

22. Verón Molinas G, Reyes AS, Zacur de Jiménez M, Mestral E de. Knowledge and practices of Pediatricians regarding perinatal care in children with malformations with a poor prognosis. Pediatr. (Asunción). 2016;43(3):220-4.

ABSTRACT. Background. Bioethics understands that any therapeutic or diagnostic proposal must define the goal to be achieved, the benefit to be obtained and the risks to be faced. It requires knowing the natural history of the disease and its complications, as well as estimating the chances of success and failure adjusted to each patient in particular. Sources: The bibliographic search was carried out using the search engines Medline, ProQuest, SCOPUS, using the keywords "ethics in the surgical act", "bioethics", "clinical ethics", "ethics in obstetrics", "therapeutic abortion", ethical considerations in pregnancy and cancer "' ethics and oncological surgery "' ethical dilemmas in anesthetic practice ", both in Spanish and English. The search was limited from 2014 to 2018. The most cited models are those included in the present review. Development: The ethical behavior should not only be limited to the surgical act, but to all the behavior of the surgeon, accepting that all their acts must be ethically valid, from the clinical study of the patient and the request of the auxiliary diagnostic studies based on a Rationale criteria up to the information given to the patient and family, the surgery and postoperative surveillance Conclusions: Every day we face ethical dilemmas so it is evident the need to continuously train in these subjects to health personnel both medical, nursing and all staff that has contact with patients in order to avoid therapeutic cruelty and achieve the maximum well-being of the patient and their family members, from an integral point of view. The main and initial ethical obligation of every doctor is to be prepared.

Keywords: Surgery, Ethics, Clinical ethics, Gynecology, Obstetrics, Unnecessary procedures. 